\title{
Host Modification and Restriction with a Mycobacteriophage Isolated from a Pseudolysogenic Mycobacterium chelonei
}

\author{
By WILBUR D. JONES, JR, AND JOSEPH GREENBERG \\ Mycobacteriology Branch, Center for Disease Control, Public Health Service, \\ U.S. Department of Health, Education, and Welfare, Atlanta, Georgia 30333, U.S.A.
}

(Received 5 August 1976; revised 25 September 1976)

\section{SUMMARY}

A pseudolysogenic Mycobacterium chelonei and its phage $\phi 630$ are described. Phage $\phi 630$ is the first mycobacteriophage reported to be resistant to the nonpolar solvents chloroform, dioxan and diethyl ether. The phage had a latent period of $75 \mathrm{~min}$, a rise period of $90 \mathrm{~min}$ and a burst size of $5 \mathrm{I}$. Evidence is presented for host modification and restriction. Phage $\phi 630 \mathrm{~A}$, grown on host strain $M$. chelonei $\mathrm{F}-630 \mathrm{Rg}$, plated on the alternative host $M$. smegmatis ATCC607 with an efficiency of plating (e.o.p.) of $10^{-5}$. Phage $\phi 630 \mathrm{~B}$, grown on host $M$. smegmatis, plated with an e.o.p. of ${ }^{-1} 0^{-5}$ on the alternative host F-630 Rg. Phages $\phi 630 \mathrm{~A}$ and $\phi 630 \mathrm{~B}$ adsorbed equally well on their alternative hosts and on their indicator host strains. The progeny of plaques from initial platings on the alternative host, when grown in the alternative host, exhibited a marked reduction in e.o.p. on their original host.

\section{INTRODUCTION}

In contrast to other phage-bacterial systems, little is known about the mycobacteriophage-mycobacterial host relationship. Bowman (1958) was the first to characterize quantitatively the virus-host relationship in the mycobacteria. Lysogens of Mycobacterium smegmatis were characterized by a high frequency of 'cured' cells in the bacterial population and a high titre of phage present in the supernatant fluids of the cultures (Jones \& White, 1968; Hubácek, Kupkova \& Mohelská; 1970, Baess, 1971). The virus-host relationship in the above systems has the characteristics of pseudolysogeny as defined by Lwoff (1953).

Methods for phage typing isolates of $M$. tuberculosis are currently being investigated in a series of co-operative studies (Rado et al., 1975). The phage typing of $M$. tuberculosis could more easily be understood and the methods more easily standardized, if some basic information were available concerning the phage-host relationships. Redmond (1963) and Marion \& Bradley (I964) suggested that phages adapted to alternative mycobacterial hosts should be used for phage typing. Nördström \& Grange (1974) used adapted phages in typing of the species $M$. ranae. Rado, Bates \& Fitzhugh (I976) and Jones \& Greenberg (1976) presented evidence suggesting that host modification and restriction of the phages plays a significant role in phage typing of $M$. tuberculosis. Because of technical problems that exist in working with $M$. tuberculosis and the time required to obtain results from such slow-growing mycobacteria, the availability of a rapidly growing mycobacterial system that could be used to develop techniques and methods and could be applied to $M$. tuberculosis would be a definite advantage (Rado et al., 1976; Jones \& Greenberg, 1976).

The present investigation describes the characteristics of a pseudolysogenic isolate of 
$M$. chelonei and its phage $\phi 630$. Evidence is presented for host modification and restriction of the phage, using $M$. smegmatis ATcc607 as the alternative host.

\section{METHODS}

Mycobacteria and phages. Mycobacterium chelonei strain F-630 $(\phi 630)$ produced plaques on a soft agar lawn of itself. If grown in $7 \mathrm{H} 9$ broth containing Tween 80 , the culture produced smooth $\mathrm{F}(\mathrm{SmF})$ and rough $\mathrm{F}(\mathrm{RgF})$ colony types in the ratio of $4:$ I when streaked on agar media for colony determination (Jones \& Kubica, 1965). After extensive single colony purification the SmF colony types continued to produce $\mathrm{RgF}$ variants and to liberate phage, whereas the purified RgF colony types were stable and did not produce phage. A single 'cured' $\mathrm{RgF}$ isolate was selected and designated as F-630 Rg. A mutant of F-630 Rg which was resistant to dihydrostreptomycin sulphate at $1000 \mu \mathrm{g} \mathrm{ml}^{-1}$ was isolated and designated as F-630 RgSMr. Mycobacterium smegmatis ATCC607, hereafter referred to as 607 , was also used as a host strain.

The phage isolated directly from $\mathrm{F}-630(\phi 630)$ and replicated on $\mathrm{F}-630 \mathrm{Rg}$ as the indicator host strain was designated as phage $\phi 630 \mathrm{~A}$. After phage $\phi 630 \mathrm{~A}$ was adapted to replicate on 607 as the indicator host strain, it was designated as phage $\phi 630 \mathrm{~B}$.

Media. Dubos oleic agar (BBL) supplemented with Dubos oleic albumin complex (Difco) (OAA agar) was used for colony determination, for colony forming unit assays, and as the hard basal medium for phage assays. The $7 \mathrm{H} 9$ broth medium for bacterial growth was Middlebrook $7 \mathrm{H}_{9}$ broth (Difco), supplemented with $0.05 \%$ (v/v) Tween 80 (Atlas Power Company) and Middlebrook ADC enrichment (Difco).

Before use in the phage tests, the bacteria grown in $7 \mathrm{H} 9$ broth were washed by centrifuging the cultures at $5000 \mathrm{rev}$. $\mathrm{min}^{-1}$ for $15 \mathrm{~min}$, resuspending the pellet in heart infusion broth (Difco) supplemented with $0.02 \mathrm{M}$-calcium chloride (HI broth), and recentrifuging. The bacterial pellets were then resuspended in tempered $\left(37^{\circ} \mathrm{C}\right) \mathrm{HI}$ broth. Phage assays were performed according to the soft agar overlay method (Adams, 1959). Soft agar overlays were prepared with Dubos broth base w/o Tween 80 (Difco) supplemented with $0.7 \%(\mathrm{w} / \mathrm{v})$ agar. The above media were all prepared and used as previously described (Jones \& White, I968). All bacterial cultures were grown at $37^{\circ} \mathrm{C}$.

Bacterial and phage assays. Assays for colony forming units (c.f.u. $\mathrm{ml}^{-1}$ ), phage assays (p.f.u. $\mathrm{ml}^{-1}$ ), adsorption tests and one-step growth tests were performed as described previously (Jones \& David, I97I). Single burst experiments were done as described by Adams (1959). Specific phage $\phi 630 \mathrm{~A}$ antisera were prepared and used as described previously (Jones \& White, I968). When the methods of Adams (1959) were used, the $K$ value of the phage antiserum was 88 .

Sensitivity to non-polar solvents. Phages $\phi 630 \mathrm{~A}$ and $\phi 630 \mathrm{~B}$ were exposed to the non-polar solvents chloroform, dioxan and diethyl ether for $10 \mathrm{~min}$, as described previously for mycobacteriophage D29 (Jones, David \& Beam, 1970), and were then plated to detect p.f.u.

Electron microscopy. Lysates of phages $\phi 630 \mathrm{~A}$ and $\phi 630 \mathrm{~B}$ were prepared and examined with a GEC/AEI EM8or electron microscope as described by Grange \& Bird (1975).

Tests for modification and restriction. The efficiencies of plating (e.o.p.) of phages $\phi 630 \mathrm{~A}$ and $\phi 630 \mathrm{~B}$ on their indicator and alternative host strains were determined by the method of Adams (1959). Adsorption tests were performed for both the indicator host strain and the alternative host strain for each phage. Changes in the lytic activity of the phages were followed by picking single plaques to both host strains. 


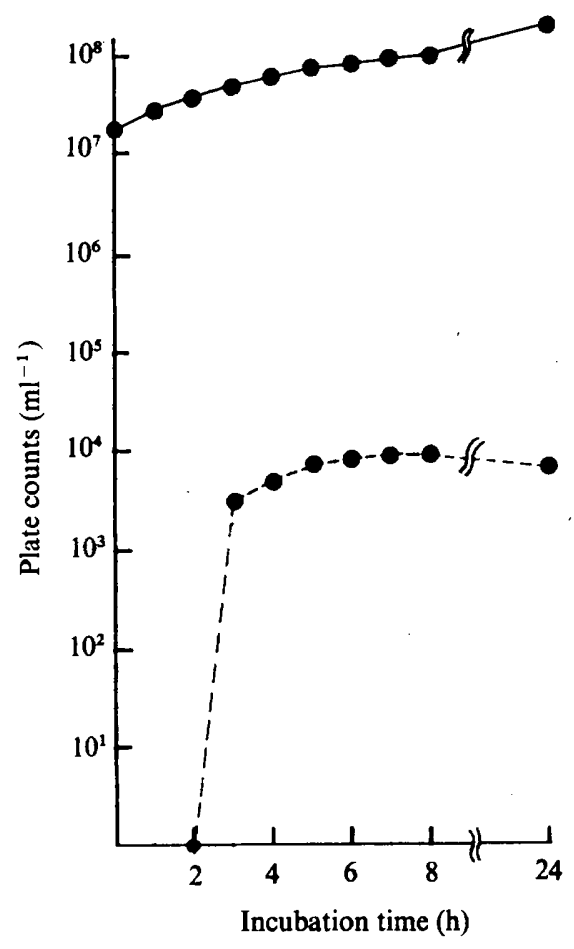

Fig. I

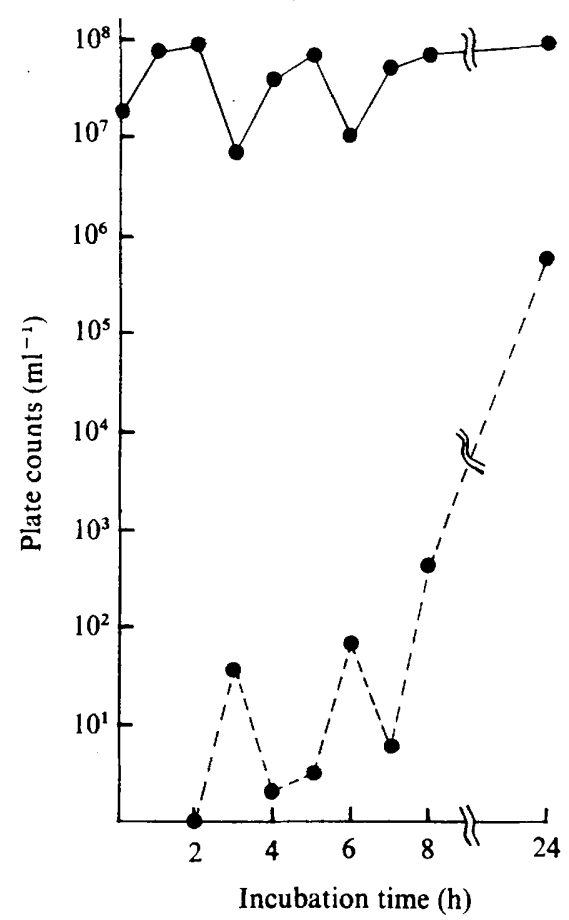

Fig. 2

Fig. I. Colony forming units (-) and plaque forming units $(---)$ produced by $M$. chelonei $\mathrm{F}-630(\phi 630)$ in heart infusion broth containing Tween 80 . Samples were removed at intervals and assayed for c.f.u. $\mathrm{ml}^{-1}$ and p.f.u. $\mathrm{ml}^{-1}$. Phage assays were done with the indicator strain $\mathrm{F}-630 \mathrm{Rg} \mathrm{SM}$ in soft agar plated on OAA agar containing dihydrostreptomycin sulphate at $1000 \mu \mathrm{g} \mathrm{ml}^{-1}$.

Fig. 2. Colony forming units $(-)$ and plaque forming units $(--)$ produced by $M$. chelonei $\mathrm{F}-630(\phi 630)$ in heart infusion broth. Other details as in legend to Fig. I.

\section{RESULTS}

Pseudolysogenic state of $\mathrm{F}-630(\phi 630)$

Since mycobacteria have a tendency to clump, the purification of single colonies is more difficult than with other bacteria. Serial transfer of single colonies had to be done at least six times on each isolate before stable $\mathrm{RgF}$ colony types that were free of phage could be obtained. The SmF colony type could not be obtained in the pure SmF form and consistently produced $\mathrm{RgF}$ colony variants and phage.

To investigate the production of phage in relation to c.f.u., a 3-day-old $7 \mathrm{H}_{9}$ broth culture of $\mathrm{F}-63 \mathrm{O}(\phi 630)$ was thoroughly washed by centrifuging five times. After each centrifugation, the bacterial pellet was resuspended in HI broth. After the final wash the culture was divided into two parts; one part contained HI broth, and the other HI broth with $0.05 \%$ Tween 80 to prevent readsorption of phage. The two cultures were incubated at $37^{\circ} \mathrm{C}$. Samples were removed at intervals and assayed for c.f.u. $\mathrm{ml}^{-1}$ and p.f.u. $\mathrm{ml}^{-1}$. The phage assays were done with $\mathrm{F}-630 \mathrm{RgSM}$ ? as the indicator host and OAA agar containing dihydrostreptomycin sulphate at $1000 \mu \mathrm{g} \mathrm{ml}^{-1}$. The data obtained for $\mathrm{HI}$ plus Tween 80 test culture are shown in Fig. I. The c.f.u. counts gradually increased over the $24 \mathrm{~h}$ test period. Phages appeared and rapidly increased in numbers between the $2 \mathrm{~h}$ and $3 \mathrm{~h}$ samplings 


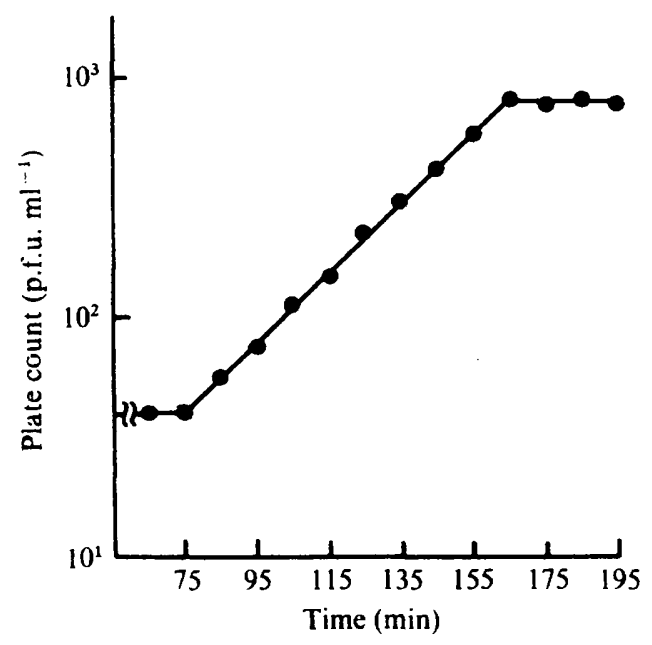

Fig. 3

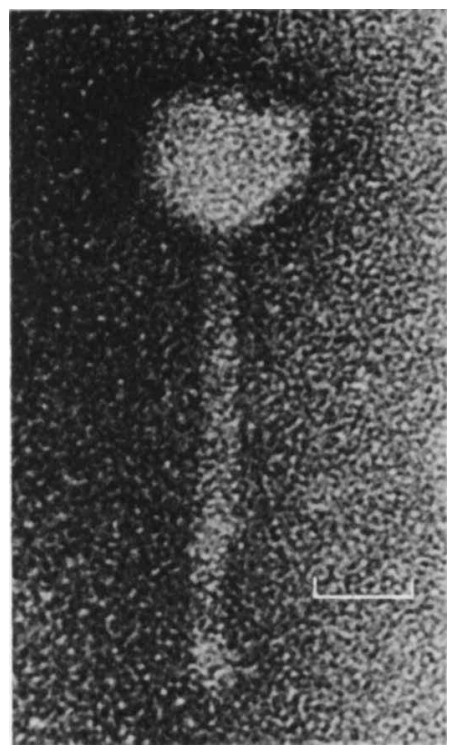

Fig. 4

Fig. 3. One-step growth curve of phage $\phi 630 \mathrm{~A}$ on its indicator host strain F- $630 \mathrm{Rg}$. Phage assays were done with strain $\mathrm{F}-630 \mathbf{R g ~ S M}^{\mathrm{r}}$ in soft agar plated on OAA agar containing dihydrostreptomycin sulphate at $1000 \mu \mathrm{g} \mathrm{ml}^{-1}$.

Fig. 4. Electron micrograph of mycobacteriophage $\phi 630 \mathrm{~A}$. Bar marker represents $40 \mathrm{~nm}$.

and continued to increase slightly over the next $4 \mathrm{~h}$. The data obtained for the HI broth test culture are shown in Fig. 2. In contrast to the culture containing Tween 80, both the c.f.u. and p.f.u. assays of the culture containing only $\mathrm{HI}$ broth fluctuated over the $24 \mathrm{~h}$ test period; however, there was a correlation between the decreases and increases in the c.f.u. and p.f.u. counts. In addition, although the number of phages released initially was smaller in $\mathrm{HI}$ broth than in HI broth plus Tween 80, the final phage titre in $\mathrm{HI}$ broth was $10^{6}$ p.f.u. $\mathrm{ml}^{-1}$, whereas in $\mathrm{HI}$ broth plus Tween 80 the final titre was only $5 \times 10^{3}$ p.f.u. $\mathrm{ml}^{-1}$.

\section{Biological properties of phages $\phi 630 \mathrm{~A}$ and $\phi 630 \mathrm{~B}$}

One-step growth tests with phage $\phi 630 \mathrm{~A}$ on its indicator host strain F-630 Rg (Fig. 3) showed that the phage had a latent period of $75 \mathrm{~min}$, followed by a rise period of $90 \mathrm{~min}$. The burst size of phage $\phi 630 \mathrm{~A}$ on F-630 Rg was $5 \mathrm{I}$. Phage $\phi 630 \mathrm{~A}$ antiserum had a $K$ value of 88 against both phages $\phi 630 \mathrm{~B}$ and $\phi 630 \mathrm{~A}$. Phages $\phi 630 \mathrm{~A}$ and $\phi 630 \mathrm{~B}$ were not inactivated by chloroform, dioxan or diethyl ether. Electron microscopy of phage $\phi 630 \mathrm{~A}$ showed a hexagonal head of approximately $60 \mathrm{~nm}$ diam. and a tail approximately I $\mathrm{nm}$ in diam. and $260 \mathrm{~nm}$ in length (Fig. 4). Phage $\phi 630 \mathrm{~B}$ was similar in morphology to phage $\phi 630 \mathrm{~A}$.

\section{Evidence for modification and restriction}

Phage $\phi 630 \mathrm{~A}$, grown on $M$. chelonei strain F-630 Rg, plated with an e.o.p. of $1 \cdot 2 \times 10^{-5}$ on $M$. smegmatis strain 607 . Plaques of phage $\phi 630 \mathrm{~A}$ which formed on strain 607 were transferred to 607 again; the phage replicated on the 607 host was designated as phage $\phi 630 \mathrm{~B}$. With the e.o.p. of phage $\phi 630 \mathrm{~B}$ on its indicator host 607 dèsignated as 1 , the e.o.p. on F-630 Rg was $\mathrm{I} \times 1 \mathrm{IO}^{-5}$. Adsorption tests were performed to determine if the low e.o.p. of 
Table I. Adsorption of phages $\phi 630 \mathrm{~A}$ and $\phi 630 \mathrm{~B}$ to their indicator and alternative host strains

Adsorption was measured by the decrease in phage titre in the supernatant fluids of samples taken at 0 and $15 \mathrm{~min}$. Results are averages of four plates per test.

\begin{tabular}{|c|c|c|c|c|c|}
\hline \multirow[b]{2}{*}{ Phage } & \multirow[b]{2}{*}{ Time (min) } & \multicolumn{2}{|c|}{ Strain 607} & \multicolumn{2}{|c|}{ Strain $\mathbf{F - 6 3 0} \mathbf{R g}$} \\
\hline & & $\ldots 0$ & 15 & 0 & I5 \\
\hline $\begin{array}{l}\phi 630 \mathrm{~A} \\
\phi 630 \mathrm{~B}\end{array}$ & & $\begin{array}{l}6.0 \times 10^{5} \\
6.0 \times 10^{5}\end{array}$ & $\begin{array}{l}3.1 \times 10^{4} \\
2.9 \times 10^{4}\end{array}$ & $\begin{array}{l}6.0 \times 10^{5} \\
6.0 \times 10^{5}\end{array}$ & $\begin{array}{l}3.0 \times 10^{4} \\
3.0 \times 10^{4}\end{array}$ \\
\hline
\end{tabular}

phage $\phi 630 \mathrm{~A}$ on 607 and phage $\phi 630 \mathrm{~B}$ on $\mathrm{F}-630 \mathrm{Rg}$ were the result of the failure of the phages to adsorb to their alternative host strains. Both phages adsorbed equally well on both host strains (Table I).

Plaques of $\phi 630 \mathrm{~A}$ on 607 and $\phi 630 \mathrm{~B}$ on $\mathrm{F}-630 \mathrm{Rg}$ were transferred using sterile toothpicks to lawns of both $\mathrm{F}-63 \mathrm{ORg}$ and 607 . A second transfer of individual plaques was made to lawns of F-630 Rg and 607 . In addition, plaques were transferred from lawns of F-630 Rg containing plaques of phage $\phi 630 \mathrm{~A}$ and lawns of 607 containing plaques of phage $\phi 630 \mathrm{~B}$. Phage $\phi 630 \mathrm{~A}$ picked directly from a lawn of F-630 Rg would not lyse 607 . Phage $\phi 630 \mathrm{~B}$ picked directly from a lawn of 607 would not lyse F-630 Rg. Surviving plaques of phage $\phi 630 \mathrm{~A}$ on lawns of 607 lysed both F-630 Rg and 607 on first transfer; however, on second transfer these phages would lyse only 607 . Surviving plaques of phage $\phi 630 \mathrm{~B}$ on F-630 Rg lysed lawns of both F-630 Rg and 607 , but on second transfer these phages would lyse only lawns of F-63o Rg.

\section{DISCUSSION}

The pseudolysogenic $M$. chelonei strain F-630( $\phi 630)$ had the same general characteristics as $M$. smegmatis ( $\phi \mathrm{D} 29$ ) reported by Jones \& White (1968). The phage-producing colonies were of the SmF colony type, and the cured, non-phage-producing colonies were of the $\mathrm{RgF}$ type. In $7 \mathrm{H}_{9}$ broth containing Tween 80 , the ratio of $\mathrm{Rg}$ to Sm colony types was $4: \mathrm{I}$. Using HI broth with and without Tween 80 , it was demonstrated that the high titre of phage in the supernatant fluids was dependent on the phage replication on the susceptible cells in the bacterial population. White \& Knight (1954) demonstrated that Tween 80 effectively blocks adsorption of the phages to their host cells. The fluctuation of the c.f.u. count and its relationship to phage adsorption and release has been described by Jones \& David (197I), who used phage D29 and $M$. smegmatis ATCC607.

Mycobacteriophages $\phi 630 \mathrm{~A}$ and $\phi 630 \mathrm{~B}$ were resistant to the nonpolar solvents chloroform, dioxan and diethyl ether. This differs from findings with other mycobacteriophages, including phage D29, which are inactivated by these solvents (Bowman, 1969; Jones et al., I970; Sellers \& Tokunaga, 1970; Bowman et al., 1973; Jones, 1973). With this exception, the biological characteristics of phages $\phi 630 \mathrm{~A}$ and $\phi 630 \mathrm{~B}$, such as burst size, one-step growth cycle, the low $K$ value of the phage antiserum and morphology, were similar to those of other mycobacteriophages.

Mycobacteriophages $\phi 630 \mathrm{~A}$ and $\phi 630 \mathrm{~B}$ plated with an e.o.p. of $10^{-5}$ on their alternative host strains. Adsorption studies demonstrated that both phages adsorbed equally well on their indicator and alternative host strains. These observations ruled out lack of adsorption as a possible explanation of the low e.o.p. on the alternative host strains. The fact that phage $\phi 630 \mathrm{~A}$ antisera neutralized phages $\phi 630 \mathrm{~A}$ and $\phi 630 \mathrm{~B}$ with equal efficiency is taken as evidence that $\phi 630 \mathrm{~A}$ and $\phi 630 \mathrm{~B}$ are the same phage. The transfer of single plaques of phage 
$\phi 630 \mathrm{~A}$ from its indicator strain $\mathrm{F}-630 \mathrm{Rg}$, and of phage $\phi 630 \mathrm{~B}$ from its indicator strain 607 , demonstrated that, under these conditions, phage $\phi 630 \mathrm{~A}$ lysed only F-630 Rg and phage $\phi 630 \mathrm{~B}$ lysed only 607 . The initial transfer of the surviving plaques of the two phages on their alternative hosts resulted in the lysis of both F-630 Rg and 607 strains; however, a second single plaque transfer resulted in the loss of lytic activity of either phage on its original indicator host strain. The capacity of the phages to adsorb to both host strains, and the loss of the lytic activity after one transfer, are characteristic of host modification and restriction (Arber \& Dussoix, 1962; Arber, 1965; Hayes, 1968). The system described here requires two transfers. To obtain modified phage plaques, the plaques must first be transferred from lawns of the alternative host containing high concentrations of plated phages. The lysis of both host strains by the phages on first transfer from the alternative host lawn is probably the result of the carry-over of both modified and unmodified phages. The first transfer serves as a purification of the modified phages which is reflected in the second step.

With the rapidly-growing system described in this report as a model system, studies are in progress to investigate host modification and restriction in more detail and the role it plays in the phage typing of mycobacteria.

We thank Dr R. G. Bird, London School of Hygiene and Tropical Medicine, for electron microscope pictures of phages $\phi 630 \mathrm{~A}$ and $\phi 630 \mathrm{~B}$, and Dr J. M. Grange, Middlesex.Hospital Medical School, London, for confirming that strain F-630 $(\phi 630)$ is Mycobacterium chelonei.

\section{REFERENCES}

ADAMs, M H. (1959). Bacteriophages. New York: Interscience.

ARBER, W. (1965). Host controlled modification of bacteriophage. Annual Review of Microbiology 19, 365-378.

ARber, W. \& Dussoix, D. (I962). Host specificity of DNA produced by Escherichia coli. I. Host controlled modification of bacteriophage $\lambda$. Journal of Molecular Biology 5, 18-36.

BAESS, 1. (1971). Report on a pseudolysogenic mycobacterium and a review of the literature concerning pseudolysogeny. Acta pathologica et microbiologica scandinavica B79, 428-434.

Bowman, B. U., JR (1958). Quantitative studies on some mycobacterial phage host systems. Journal of Bacteriology 76, 52-62.

Bowman, B. U., JR (1969). Effect of chloroform on mycobacteriophages. Microbios 4, 347-352.

-Bowman, B. U., Newman, H. A. I., Moritz, J. M. \& Koehler, R. M. (1973). Properties of mycobacteriophage DS6A. II. Lipid composition. American Review of Respiratory Diseases 107, 42-49.

Grange, J. M. \& BIRD, R. G. (I975). The nature and incidence of lysogeny in Mycobacterium fortuitum. Journal of Medical Microbiology 8, 21 5-223.

HAYES, W. (1968). The Genetics of Bacteria and Their Viruses. New York: John Wiley.

Húb́cek, J., Kupkova, H. \& MohelskÁ, H. (1970). A special case of lysogeny in Mycobacterium avium. Folia microbiologica 15, 341-346.

JONEs, W. D., JR (1973). Studies on the bacteriophage of a naturally lysogenic Mycobacterium fortuitum. American Review of Respiratory Diseases 108, 1438 - I44I.

Jonts, W. D., JR \& DAVID, H. L. (1971). Inhibition by rifampin of mycobacteriophage D29 replication in its drug resistant host, Mycobacterium smegmatis ATCC 607. American Review of Respiratory Diseases ro3, 618-624.

JoNes, W. D., JR \& GREenberg, J. (1976). Use of phage F- $\phi$ WJ-I of Mycobacterium fortuitum to discern more phage types of Mycobacterium tuberculosis. Journal of Clinical Microbiology 3, 324-326.

JONES, W. D., JR \& KUBICA, G. P. (1965). Differential colonial characteristics of mycobacteria on oleic acid-albumin and modified corn meal agars. 2. Investigation of rapidly-growing mycobacteria. Zentralblatt für Bakteriologie, Parasitenkunde, Infektionskrankheiten und Hygiene (Abteilung I) 196, 53-67.

JONES, W. D. JR \& WHITE, A. (1968). Lysogeny in mycobacteria. I. Conversion of colony morphology, nitrate reductase activity, and Tween 80 hydrolysis of Mycobacterium sp. ATCC 607 associated with lysogeny. Canadian Journal of Microbiology 14, 55 I-555.

Jones, W. D., JR, DAVID, H. L. \& BeAM, R. E. (1970). The occurrence of lipids in mycobacteriophage D29 propagated in Mycobacterium smegatis ATCC 607. American Review of Respiratory Diseases 102, 814-817.

LwoFf, A. (1953). Lysogeny. Bacteriological Reviews 17, 269-337. 
MARION, R. E. \& BRADLEY, S. G. (1964). Derivation of new mycobacteriophage typing reagents by propagation on alternate hosts. American Review of Respiratory Diseases 89, 674-676.

NÖRDSTRÖM, G. \& GRANGE, J. M. (1974). Bacteriophage typing of Mycobacterium ranae (fortuitum). The use of unadapted and adapted phages in the development of a typing system. Acta pathologica et microbiologica scandinavica $\mathrm{B82}, 87-93$.

Rado, T. A., Bates, J. H., Engel, H. W. B., Mankiewicz, E., Murohashi, T., Mizuguchi, Y. \& Sula, L. (1975). World Health Organization studies on bacteriophage typing of mycobacteria. Subdivision of the species Mycobacterium tuberculosis. American Review of Respiratory Diseases 111, 459-468.

Rado, T. A., Bates, J. H. \& FitzhUGH, J. K. (1976). Host controlled restriction and modification of bacteriophage in Mycobacterium tuberculosis. Journal of General Virology 30, 91-97.

Redmond, W. B. (1963). Bacteriophages of the mycobacteria - a review. Advances in Tuberculosis Research 12, $191-229$.

Sellers, M. I. \& TokunaGa, T. (1970). Inactivation of mycobacteriophages by lipid solvents. In HostVirus Relationships in Mycobacteria, Nocardia and Actinomyces, pp. 134-143. Springfield, Illinois: Charles C. Thomas.

WhITE, A. \& KNIGHT, V. (1954). Effect of Tween ${ }^{R} 80$ and serum on the interaction of mycobacteriophage D29 with certain mycobacterial species. American Review of Respiratory Diseases 77, $134-145$. 